\title{
A DELPHI study on aspects of study design to overcome knowledge gaps on the burden of disease caused by serogroup $B$ invasive meningococcal disease
}

Ole Marten ${ }^{1 *+} \mathbb{D}$, Florian Koerber ${ }^{2,18+}$, David Bloom³, Monika Bullinger ${ }^{4}$, Corinne Buysse ${ }^{5}$, Hannah Christensen ${ }^{6}$, Philippe De Wals ${ }^{7}$, Christian Dohna-Schwake ${ }^{8}$, Philipp Henneke ${ }^{9}$, Markus Kirchner ${ }^{2}$, Markus Knuf ${ }^{10,11,}$

Burkhard Lawrenz ${ }^{12,13}$, Andrea L. Monteiro ${ }^{14,19}$, Joseph Patrick Sevilla ${ }^{15}$, Nicolas Van de Velde ${ }^{16}$, Robert Welte ${ }^{2}$, Claire Wright ${ }^{17}$ and Wolfgang Greiner ${ }^{1}$

\begin{abstract}
Background: Value assessment of vaccination programs against serogroup B invasive meningococcal disease (IMD) is on the agenda of public health authorities. Current evidence on the burden due to IMD is unfit for pinning down the nature and magnitude of the full social and economic costs of IMD for two reasons. First, the concepts and components that need to be studied are not agreed, and second, measures of the concepts that have been studied are weak and inconsistent. Thus, the economic evaluation of the available serogroup B meningococcal (MenB) vaccines is difficult. The aims of this DELPHI study are to: (1) agree on the concepts and components determining the burden of MenB diseases that need to be studied; and (2) seek consensus on appropriate methods and study designs to measure quality of life (QoL) associated with MenB induced long-term sequelae in future studies.
\end{abstract}

Methods: We designed a DELPHI questionnaire based on the findings of a recent systematic review on the QoL associated with IMD-induced long-term sequelae, and iteratively interviewed a panel of international experts, including physicians, health economists, and patient representatives. Experts were provided with a controlled feedback based on the results of the previous round.

Results: Experts reached consensus on all questions after two DELPHI rounds. Major gaps in the literature relate (i) to the classification of sequelae, which allows differentiation of severity levels, (ii) to the choice of QoL measures, and (iii) to appropriate data sources to examine long-term changes and deficits in patients' QoL.

Conclusions: Better conceptualisation of the structure of IMD-specific sequelae and of how their diverse forms of severity might impact the QoL of survivors of IMD as well as their family network and care-providers is needed to generate relevant, reliable and generalisable data on QoL in the future. The results of this DELPHI panel provide useful guidance on how to choose the study design, target population and appropriate QoL measures for future research and hence, help promote the appropriateness and consistency in study methodology and sample characteristics.

Keywords: Quality of life, Meningococcal disease, Neisseria meningitidis, DELPHI

\footnotetext{
*Correspondence: ole.marten@uni-bielefeld.de; ole.marten@uni-bielfeld.de

${ }^{\dagger}$ Ole Marten and Florian Koerber are co-first authors

'Department of Health Economics and Health Care Management, School of

Public Health, Bielefeld University, Universitätsstr. 25, 33615 Bielefeld,

Germany

Full list of author information is available at the end of the article
}

(c) The Author(s). 2019 Open Access This article is distributed under the terms of the Creative Commons Attribution 4.0 International License (http://creativecommons.org/licenses/by/4.0/), which permits unrestricted use, distribution, and reproduction in any medium, provided you give appropriate credit to the original author(s) and the source, provide a link to the Creative Commons license, and indicate if changes were made. The Creative Commons Public Domain Dedication waiver (http://creativecommons.org/publicdomain/zero/1.0/) applies to the data made available in this article, unless otherwise stated. 


\section{Focus on the patient}

\section{What is the context?}

- Invasive meningococcal disease (IMD) has the highest incidence rate in young children. In the short term, IMD causes meningitis and/or sepsis. In the long term, IMD survivors can suffer from sequelae of various types and severity levels.

- Current available evidence does not fully capture the burden of long-term sequelae in IMD survivors and their societal consequences, mainly due to gaps and weaknesses in methods of assessment.

\section{What is new?}

- A DELPHI questionnaire was designed and submitted to a board of international experts, including medical doctors, health economists and patient representatives.

- The experts reached consensus on optimal and recommended sequelae classification, study sample characteristics, study design, and quality of life measurement instruments and indicators.

\section{What is the impact?}

- The experts' recommendations should help harmonise methodologies to assess the quality of life of IMD survivors (as well as their close family members and carers) with long-term sequelae in future studies so that more consistent data can be meaningfully compared, combined, and utilised.

- High-quality and relevant data are a prerequisite to support evidence-based public health decision making.

\section{Background}

Invasive meningococcal disease (IMD) potentially leads after an acute phase to serious long-term sequelae and complications and possibly premature death, thus imposing a high burden onto the patients and their carers [1-3]. The disease is caused by the bacterium Neisseria meningitidis and predominantly affects infants and young children $[4,5]$. Main clinical presentations of IMD are sepsis and/or meningitis. Six serogroups A, B, C, W, X and Y primarily cause IMD $[5,6]$. Although IMD occurs globally, the distribution of serogroups varies across regions. While serogroups $\mathrm{B}, \mathrm{C}$ and $\mathrm{Y}$ are the leading cause of IMD in Europe and other regions of higher-income, such as North America, serogroups $\mathrm{A}, \mathrm{C}, \mathrm{W}$ and $\mathrm{X}$ are dominant in the meningitis belt of sub-Saharan Africa. However, serogroup W is now also a leading cause of IMD in some European countries such as the United Kingdom or the Netherlands [7-11].

The availability of vaccines and the implementation of universal mass vaccination (UMV) programs for children decreased the incidence of serogroup $C$ in Europe $[4,11]$. Decision-making processes on whether to recommend serogroup B meningococcal (MenB) UMV in European countries are concluded or ongoing [12]. Of the reported
IMD cases in Europe in 2016, the majority (54\%) was attributable to serogroup B, whereas C accounted for $16 \%$. While serogroup $B$ is responsible for most cases of IMD, especially in children under the age of five, serogroups $C$, $\mathrm{Y}$ and $\mathrm{W}$ are predominantly responsible for IMD cases in older age groups [4]. Of note, the clinical presentation of IMD caused by serogroup B is not significantly different from that of other serogroups $[13,14]$. Further, associations between both the chance of occurrence and severity of sequelae and serogroups are inconclusive due to low case numbers $[13,15,16]$.

Although the incidence of MenB-related IMD is relatively low, the disease has high social and economic costs $[17,18]$. However, current evidence on the disease burden does a poor job of pinning down the nature and magnitude of the full costs of IMD for two reasons. First, the concepts and components that need to be studied are not agreed upon, and second, measures of the concepts that have been studied are weak and inconsistent. Hence, a proper translation of the burden on an outcome measure that can be applied for economic evaluation is lacking.

Olbrich et al. (2018) argue that the quality of life (QoL) loss caused by IMD cannot precisely be quantified and assessed since published studies are highly heterogeneous regarding the study design and QoL measurement. This is due to a lack of QoL measurements which differentiate between survivors of IMD with and without sequelae, or QoL measurements specific to types and severity levels of sequelae associated with IMD [16]. Gasparini et al. highlight the importance of accurately estimating the incidence of disease and its consequences, i.e. the type and probability of being left with certain sequelae and the corresponding disutility, to correctly account for the decrease in QoL in IMD patients [19].

Moreover, the measurement of QoL in infants and children is methodologically more challenging than in adults, as shown by Herdman et al. (2016) for IMD cases [20]. Additionally, the perception of health and impairments due to illness might change when patients age and reach adulthood, implying further requirements when QoL is measured along the transition to adulthood. This relates especially to the choice of an appropriate valuation technique, the resulting value sets or the necessity to change the instrument [21]. The impact of the various forms of sequelae on the QoL of IMD survivors remains unclear and seems underestimated $[8,16]$. Not only does IMD have a direct impact on the patients, it also has indirect consequences for carers, family members and for the society. These wider health effects might also need to be taken into account for economic evaluation [2, 3, 19].

The aims of this DELPHI study were to:

(1) agree on the concepts and components determining the burden of MenB diseases that need to be studied; and 
(2) seek consensus on appropriate methods and study designs to measure the QoL associated with MenBinduced long-term sequelae in future studies.

\section{Methods}

We used a DELPHI panel approach to find consensus on data gaps and the most appropriate study design to measure the QoL associated with IMD sequelae. The purpose of a normative DELPHI approach is to identify and answer questions of what should be and what is desirable in the future, rather than to assess the current, highly imperfect literature [22]. The conventional DELPHI aims at gathering expert opinion and studying its evolution across iterative rounds of discussion, typically gravitating toward a consensus-based group opinion among the participating experts [23].

The iterative process inherent in the DELPHI technique enables the evolution of the experts' opinion on the specified research topic, where, due to the controlled feedback, experts re-consider and re-assess their perspective by including broader views and additional information provided by the other participating experts [24, 25]. Furthermore, the DELPHI allows the application of both qualitative and quantitative measures. The former strengthens the in-depth understanding of the expert's answers, whereas the latter provides a ground for statistical analyses of the data to reach a consensus [26, 27].

\section{The expert panel}

In accordance with the expert definitions provided by Keeney et al. (2001), we invited specialists with experience in measuring QoL, especially that associated with meningococcal long-term sequelae [28]. The expert panel comprised epidemiologists, clinicians, paediatricians, psychologists, patient representatives as well as health economists. Each of the experts has profound experience with study designs, data collection, or clinical experience in the treatment of IMD or in the measurement of QoL, especially in children and adolescents. Additionally, we selected experts to cover the patient's perspective as well as clinical aspects specific to IMD. Experts were from the UK, USA, Germany, Australia, the Netherlands, Canada, Brazil and Norway in order to cover the international interest to patients, public health authorities and clinicians. Ultimately, 16 experts participated in the DELPHI panel ensuring a broad and appropriate pooling of expert opinions [25, 27].

\section{The DELPHI process}

The first DELPHI round was informed by a systematic literature review. The initial questionnaire provided to experts covered the relevant gaps identified in the recent review by Olbrich et al. [16] and comprised 11 questions (Additional file 1). Of those, five questions were formulated as choice tasks, where the experts had to choose one of the pre-defined items. Four ranking tasks asked the respondents to rank $n$ given items numerically, where 1 represents the most important and $n$ the least important option. Hence, increasing rank numbers correspond to less preferred answers [29]. Two qualitative questions were included to further explore the experts' opinions. The choice and ranking tasks gave experts the opportunity to explain their answer or to supplement the list of items within each task.

Along with the initial questionnaire, experts received in March 2017 instructions, a glossary, and a detailed introduction providing a summary of the results from the systematic literature review to ensure proper understanding of the questionnaire. The experts answered the first round questionnaire individually (via e-mail) and anonymously not knowing of the other experts, which Dalkey et al. (1962) recommend as a strategy to minimise group-based interactions between experts [23].

The second round of the DELPHI panel was held in a presence meeting in March 2017 enabling the provision of a summary of the first-round responses with a subsequent discussion giving each expert the opportunity to voice or revise his/her previous answers. Subsequently, an adapted questionnaire with eight questions was given to the experts. For the second round, the two qualitative questions 4 and 11 were dropped for parsimony, since potential answers were collected within the first round or recorded and debated during the discussion. We further dropped question 6, which was unanimously answered during the first round and, hence, a consensus on that specific question was already reached. This second questionnaire also provided feedback from the first round. For the choice tasks the answer distribution was given. Regarding the ranking tasks, experts were provided with the group ranking derived from the rank sum of each item per question.

\section{Analysis, convergence and consensus}

All closed questions had to be either ranked or selected by the experts. Descriptive analyses of the results of the ranking and choice tasks were undertaken. We aggregated the individual ranks into a group ranking by calculating the rank sums; since lower numbers represent a better rank, the items with the lowest rank sum will be considered as the preferred options. The minimum and maximum rank, the mean rank, and the mode of each item were analysed (not reported). Additionally, we evaluated the results from the choice tasks with the help of histograms inspecting the frequency distribution of the items for each question.

Convergence was measured using Kendall's W, a non-parametric test assessing the level of agreement in an expert group. The test statistic ranges from 0 to 1 , 
where 0 means no agreement and 1 represents full agreement among the experts [30]. The convergence in the choice tasks was assessed by comparing the share of experts selecting a given answer category across rounds. Hence, if the experts' opinion converges towards a mutually accepted option, the share of experts choosing this particular option should increase while the shares for the remaining items decrease.

A consensus was defined differently for rank and choice questions. In terms of the choice tasks, a consensus was reached when $2 / 3$ of the experts agreed on addressing an issue in a certain way and no veto was raised [31]. A veto could be raised due to ethical, legal, or methodological concerns. In the ranking exercises, we considered the top 3 items per question as the consensus solution, if the 3 corresponding items remained constant across the two rounds. Further, we required Kendall's W to increase from the first to the second round, implying an increased agreement among the raters [30]. In terms of Kendall's W, the level of consensus was defined as strong for $W>=0.7$, moderate for $W=0.5$ and weak for $W<0.3$ [29].

\section{Results}

We present key results of the two DELPHI rounds conducted in this study from 5 questions. The first-round questionnaire can be accessed in the Additional file 1.

We achieved an expert consensus on all ranking and choice tasks. From the first to the second round of ranking tasks we observed Kendall's W to be monotonically increasing (Tables 2, 3 and 4), resulting in moderate to strong levels of agreement after the second round. For all but one of the choice tasks a $2 / 3$ majority was achieved after the second round.

\section{Question 1 - conceptualisation of sequelae}

Table 1 tabulates responses to question 1 . For the three tier options presented, the first-round votes showed a slight preference for tier three (with 8 votes) compared to tier two with 6 votes, and tier three with 2 votes. At the end of the second round, 13 out of 14 experts voted for tier three indicating an agreement level of almost 93\%. During the process, the experts' comments emphasized the importance of

- a conceptual model of sequelae, with

$O$ a detailed classification of sequelae,

$O$ allowing to distinguish the severity of sequelae,

$\bigcirc$ reflecting the impact of multiple simultaneous sequelae

- putting in place the processes for measuring sequelae.

Experts also stated that the sought-after concept of sequelae must be meaningfully measurable and therefore requires a sufficiently large number of patients for each category.
Table 1 First and second round responses to Question 1

\begin{tabular}{lll}
\hline Tier & $\begin{array}{l}\text { Frequency } \\
\text { 1st round }\end{array}$ & $\begin{array}{l}\text { Frequency } \\
\text { 2nd round }\end{array}$ \\
\hline 3rd tier & 8 & 13 \\
2nd tier & 6 & 1 \\
1st tier & 2 & 0 \\
\hline
\end{tabular}

\section{Question 2 - target population for QoL measurement}

The results of this ranking task showed good ability to distinguish among the options (Tables 2 and 3). Assuming that all options were equally important for the disease burden assessment, the rank sums of all options would be equally high. However, the difference in the rank sums between the target groups being deemed the most and least important by the experts for a full disease burden assessment ranged from 50 to 62 points in the two younger age groups and from 65 to 72 points in the adult group. Thus, indicating that the experts had a clear preference order over the importance of the individual target groups. The first-round votes showed a strong preference, in both minor as well as severe sequelae and in all age groups, for patients with an IMD history. Further, the closest carer (i.e. parents, spouse or siblings) were important when assessing the impact of IMD on QoL. This pattern was confirmed in the second round. The preference for the response options "patient", "parents", "siblings" and "spouse" got stronger, as the rank sums decreased. The experts agreed that besides measuring the QoL losses in patients, negative spillover effects onto the patients' parents and siblings should also be considered mainly for the two younger age groups (0-5 and 6-18 year olds), whereas in the adult group (> 18 years) the spouse was more important relative to the other groups for the assessment of QoL losses. This observation is confirmed by the monotonic increase in Kendall's W indicating a strong level of agreement with $\mathrm{W}$ ranging from $0.73-0.77$ for the two younger age groups, whereas the agreement remains at a moderate level in the adult age groups (Tables 2 and 3 ).

\section{Question 7 - viable measures for QoL assessment}

The first-round votes resulted in heterogeneous responses and low agreement (Kendall's $W<0.3$ ) on using any of the presented methods to measure the QoL for the two IMD patient age groups (Table 4). However, there was a slight preference for using established measures for both age groups ( $8-18$ years and $>18$ years), i.e. existing generic or disease-specific instruments as well as the visual analogue scale. During the second round (Table 4), the preference for these measures became stronger. By contrast, methods of direct preference elicitation, where respondents are asked to attach a value to their health state directly, were ranked lowest independently of the patient's age, except for 
Table 2 Ranking results from Question 2 - light forms of sequelae

\begin{tabular}{|c|c|c|c|c|c|c|}
\hline \multirow{3}{*}{$\begin{array}{l}\text { Age group } \\
\text { Group }\end{array}$} & \multicolumn{2}{|l|}{$0-5$ years } & \multicolumn{2}{|l|}{$6-18$ years } & \multicolumn{2}{|l|}{$>18$ years } \\
\hline & \multicolumn{2}{|c|}{ Rank sum (group ranking) } & \multicolumn{2}{|c|}{ Rank sum (group ranking) } & \multicolumn{2}{|c|}{ Rank sum (group ranking) } \\
\hline & First round & Second round & First round & Second round & First round & Second round \\
\hline Patients with an IMD history & $22(1)$ & $15(1)$ & $16(1)$ & $14(1)$ & $16(1)$ & $14(1)$ \\
\hline Spouse & $\mathrm{n} / \mathrm{a}$ & $\mathrm{n} / \mathrm{a}$ & $\mathrm{n} / \mathrm{a}$ & $\mathrm{n} / \mathrm{a}$ & $46(2)$ & $37(2)$ \\
\hline Parents & $28(2)$ & $27(2)$ & $31(2)$ & $28(2)$ & $48(3)$ & $39(3)$ \\
\hline Siblings & $50(3)$ & $43(3)$ & $51(3)$ & $43(3)$ & $63(4)$ & $55(4)$ \\
\hline Peers (e.g. class mates, friends) & $69(4)$ & $60(4)$ & $63(4)$ & $57(4)$ & $66(5)$ & $60(5)$ \\
\hline Teachers & $71(5)$ & $66(5)$ & $69(5)$ & $68(5)$ & $88(6)$ & $78(6)$ \\
\hline Health Care Professionals & $72(6)$ & $71(6)$ & $78(6)$ & $72(6)$ & $88(6)$ & $79(7)$ \\
\hline Kendall's W & 0.56 & 0.74 & 0.63 & 0.77 & 0.54 & 0.60 \\
\hline
\end{tabular}

those using a visual analogue scale. The agreement level on the ranking of the alternatives reached a moderate level after the second round. During the discussion, experts raised requirements for the questionnaire. It should be cross-country validated, age adequate, and cross-study comparable. Consequently, it was concluded that either of the first to third ranked measures is a sensible choice. Alternatively, developing a disease-specific and age adequate instrument should be considered.

\section{Question 9 - demand for more longitudinal data}

Figure 1 summarises the responses given during both rounds. During the first DELPHI round, 9 experts voted for a longitudinal design, whereas 7 experts chose a cross-sectional design. There was also agreement that $2-3$ repetitions of the survey were sufficient, and there was almost equal opinion on the follow-up period: 3-20 years with a mode of 5 years. Two experts noted that cross-sectional surveys might be sufficient given that the conduct of long-term longitudinal studies is difficult and costly. Nonetheless, they preferred a longitudinal design. The distinction between studies using the same or different sample(s) for a repeated cross-sectional study was not considered during the 1st round. Given the preference for a longitudinal study design, this option was made available to address this characteristic. The results from the second round show a shift from a longitudinal study to a cross-sectional design, as all participants voted for a cross-sectional design. Finally, the experts agreed that the minimum requirement for a disease burden study would be a single cross-sectional design (67\% of experts). They also indicated that a repeated cross-sectional study would be preferred.

\section{Question 10 - choice of an observational study design}

During the first round, 4 experts selected Cohort study, 6 selected Case-control study, and 6 selected Cross-sectional study. It should be noted that the case-control design was defined as a retrospective comparison of health status between patients with a history of IMD (cases) and no such history (controls). Thus, after the first round, a tendency towards pragmatic and less costly study designs was observed. In the meeting prior to the second round, this question was discussed more in depth and in the light of the preceding question 9. In the second round, eight experts voted for a cross-sectional design, while 6 experts preferred a case-control study and 2 experts chose the cohort study design, hence with $50 \%$ of the respondents

Table 3 Ranking results from Question 2 - severe forms of sequelae

\begin{tabular}{|c|c|c|c|c|c|c|}
\hline \multirow{3}{*}{$\begin{array}{l}\text { Age group } \\
\text { Group }\end{array}$} & \multicolumn{2}{|l|}{$0-5$ years } & \multicolumn{2}{|l|}{$6-18$ years } & \multicolumn{2}{|l|}{$>18$ years } \\
\hline & \multicolumn{2}{|c|}{ Rank sum (group ranking) } & \multicolumn{2}{|c|}{ Rank sum (group ranking) } & \multicolumn{2}{|c|}{ Rank sum (group ranking) } \\
\hline & First round & Second round & First round & Second round & First round & Second round \\
\hline Patients with an IMD history & $22(1)$ & $14(1)$ & $17(1)$ & $14(1)$ & $16(1)$ & $14(1)$ \\
\hline Spouse & $\mathrm{n} / \mathrm{a}$ & $\mathrm{n} / \mathrm{a}$ & $\mathrm{n} / \mathrm{a}$ & $\mathrm{n} / \mathrm{a}$ & $42(2)$ & $37(2)$ \\
\hline Parents & $27(2)$ & $28(2)$ & $29(2)$ & $28(2)$ & $44(3)$ & $38(3)$ \\
\hline Siblings & $49(3)$ & $42(3)$ & $49(3)$ & $42(3)$ & $62(4)$ & $55(4)$ \\
\hline Peers (e.g. class mates, friends) & $68(4)$ & $60(4)$ & $65(4)$ & $57(4)$ & $70(5)$ & $60(5)$ \\
\hline Teachers & $72(5)$ & $64(5)$ & $67(5)$ & $66(5)$ & $86(6)$ & $79(6)$ \\
\hline Health Care Professionals & $73(6)$ & $71(6)$ & $78(6)$ & $72(6)$ & $88(6)$ & $81(7)$ \\
\hline Kendall's W & 0.37 & 0.73 & 0.39 & 0.75 & 0.57 & 0.64 \\
\hline
\end{tabular}


Table 4 Ranking results to Question 7

\begin{tabular}{|c|c|c|c|c|}
\hline \multirow{3}{*}{$\begin{array}{l}\text { Age group } \\
\text { Method }\end{array}$} & \multicolumn{2}{|l|}{$8-18$ years } & \multicolumn{2}{|l|}{$>18$ years } \\
\hline & \multicolumn{2}{|c|}{ Rank sum (group ranking) } & \multicolumn{2}{|c|}{ Rank sum (group ranking) } \\
\hline & First round & Second round & First round & Second rounc \\
\hline Using an existing generic instrument & $33(1)$ & $23(1)$ & $45(1)$ & $22(1)$ \\
\hline Using an existing disease-specific questionnaire & $34(2)$ & $34(2)$ & $48(2)$ & $34(2)$ \\
\hline Direct preference elicitation using a Visual Analogue Scale & $44(3)$ & $46(3)$ & $48(2)$ & $50(3)$ \\
\hline Using a Discrete Choice Experiment & $59(5)$ & $63(5)$ & $60(4)$ & $59(5)$ \\
\hline Direct preference elicitation using the Time trade-off & $74(7)$ & $81(7)$ & $64(5)$ & $83(7)$ \\
\hline Direct preference elicitation using the Standard Gamble & $70(6)$ & $75(6)$ & $65(6)$ & $75(6)$ \\
\hline Develop a new disease specific questionnaire & $49(4)$ & $50(4)$ & $66(7)$ & $56(4)$ \\
\hline Kendall's W & 0.23 & 0.49 & 0.07 & 0.50 \\
\hline
\end{tabular}

voting for a cross-sectional design no $2 / 3$ majority for a specific observational study design was reached.

The experts reasoned that in a cohort study the evolution of sequelae could be assessed; however, it would be time-consuming and expensive. By comparison, a case-control study as defined above addresses the rarity and long latency of IMD and its corresponding sequelae, allows comparison against healthy controls and covers a variability of patients. Nevertheless, case-control studies are prone to bias. Finally, a cross-sectional study can be supplemented with retrospective data, would also allow to include a control group, is easy to conduct, inexpensive and therefore most practical. No concerns about direction of causality were raised.

\section{Discussion}

IMD is an uncommon, but severe disease. MenC UMV programs in Europe helped decrease the MenC related disease burden. While MenB is still responsible for the majority of IMD cases in Europe, respective UMV programmes are currently implemented less frequently in European countries. Studies evaluating the cost-effectiveness of uncommon but severe diseases such as MenB are challenging and draw on incomplete evidence on the actual disease burden for this particular serogroup [8, 19].

Our results reinforce the need for a more detailed examination of sequelae attributable to IMD and the direct long-term consequences for patients' QoL as well as the indirect impact onto their parents, siblings or spouse. Experts argued that a detailed classification of sequelae should distinguish both the severity of sequelae and the impact of having multiple simultaneous sequelae. The experts' perception of an unclear structure of sequelae accords with the findings of a recent literature review on this topic, which reports considerable heterogeneity in prevalence rates and inconsistent sampling

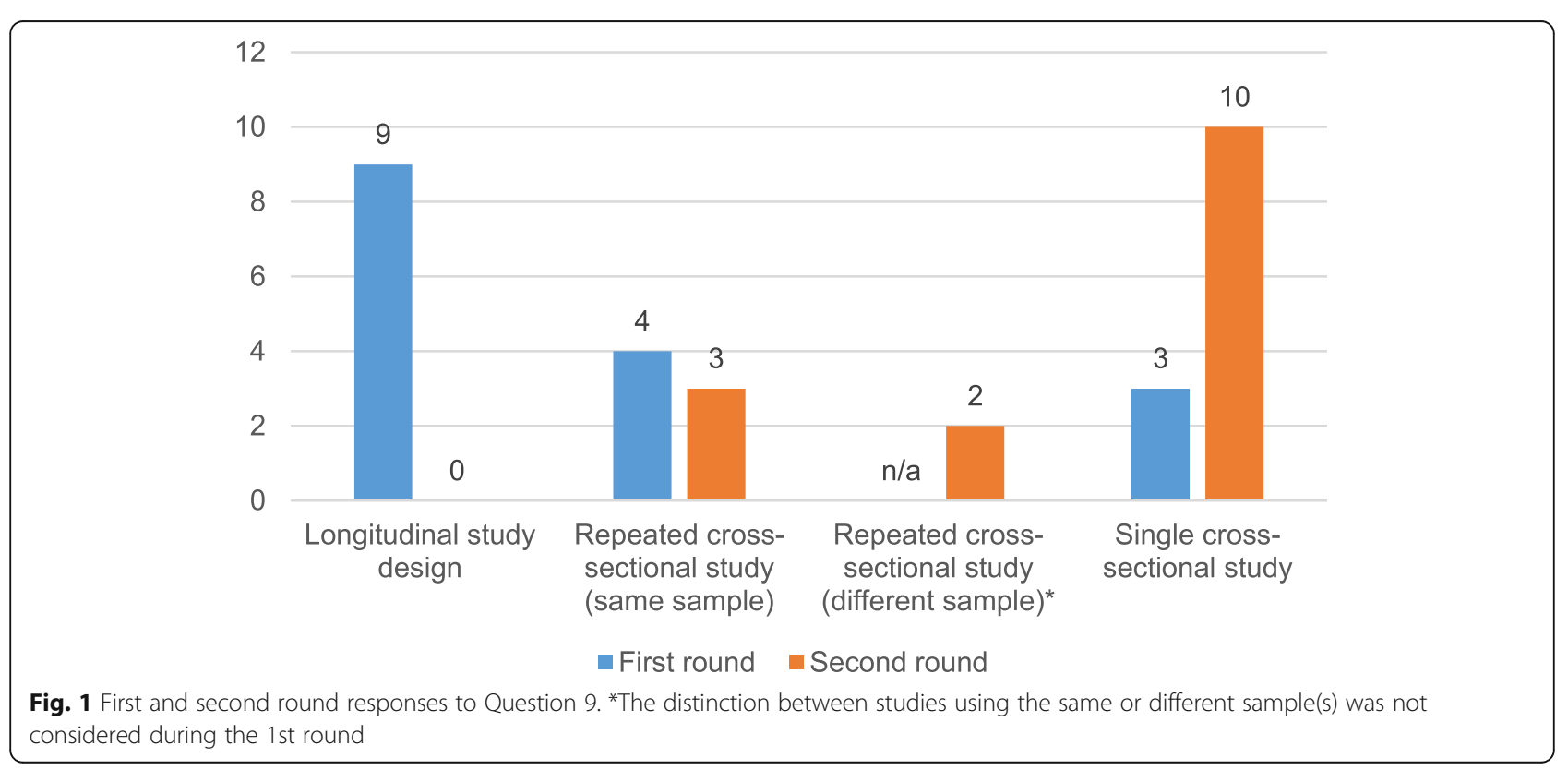


strategies [16]. The experts emphasised that a valid operationalisation of the postulated classification is a pre-requisite for a comprehensive QoL measurement. Similarly, Viner et al. (2012) argued that the lack of reliable QoL data prevents the correct assessment of disease burden associated with IMD [8].

On the other hand, the sought-after classification of sequelae should also be pragmatic, since highly stratified patient samples cannot be achieved in future prospective studies due to the small number of patients worldwide as well as the rarity of some sequelae. For example, in 2015 only 1682 prevalent serogroup B IMD cases were reported in countries of the European Union [12].

Generally, the expert consensus on the remaining questions of this DELPHI study suggests pragmatic and goal-driven methods for future studies to overcome the current lack of knowledge, which is impeding rational decisions on the allocation of resources to vaccine development and delivery. The experts acknowledged budget and practical constraints in their answers, and therefore preferred a single cross-sectional study. Further, it was argued that a strategic sampling approach, targeting patients who contracted the disease at different times, as well as the inclusion of a control group might help to examine the evolution of sequelae and to assess QoL losses. However, with respect to the study design we did not achieve a $2 / 3$ majority for a specific design; nonetheless, a tendency towards a retrospective data study with a control group design was observed.

The preference for existing generic and disease-specific QoL measures also reflects pragmatism. However, it should be acknowledged that disease-specific QoL measures are problematic, since they usually do not allow comparisons across different disease areas. They either need to be preference-based or mapped onto a generic preference-based measure in order to be comparable across disease areas or to produce utilities for use in cost-utility or modelling studies, which was desired by the experts. Further, the choice of an appropriate QoL measure is constrained by decision-making bodies in several countries, such as the National Institute for Health and Care Excellence (NICE) in the United Kingdom [32].

Moreover, if the aim is to measure QoL across different age groups, as it is necessary for IMD cases, the assessment of health states along the transition to adulthood is complicated. For this purpose, several established and child-specific QoL measures were discussed. To date, standard paediatric QoL measures, such as the EQ-5D-Y [33] or the Child Health Utility 9D (CHU9D) [34], suffer from at least one of three key limitations. First, validation for the youngest age group is still pending; second, comparability with QoL questionnaires for adults is limited; and third, the scoring mechanisms to translate the children's answers into a utility score might only be available from adult populations. While the EQ-5D-Y does not offer a child specific value set yet, the EQ-5D-Y broadens the applicable age range (4 to 17 years) and enables the comparison with the instrument for adults by using the same descriptive system. In this sense, the EQ-5D [35] could be applied, as the instruments offer some methodological advantages over other paediatric instruments [21].

Since IMD mainly affects children and adolescents, negative spillover effects on family members and carers must be considered in a comprehensive disease burden study. Our results suggest that, as a practical matter, these primarily need to be considered for the family network and carers, i.e. the parents or siblings of patients $\leq 18$ years. The expert panel agreed that the relevance of the closest relatives and carer, i.e. the parents or the spouse, spans all the included age groups and severity levels. Our conclusion accords with findings from Al-Janabi et al. (2016), who found the most impactful health spillovers in close family members, but with a declining rate with increasing social distance to the patient [2]. Thus, the targeted group in a future IMD-related QoL study should at least consist of patients and their closest relatives and carer, irrespective of the severity of sequelae and age of the patient.

Our DELPHI study of an expert panel with an international composition and multi-disciplinary experience yielded robust results showing a high level of consensus after the second round, reaching a moderate to strong level of agreement on ranking tasks and a majority of $2 /$ 3 in choice tasks, except for one question. The structure of our study combined the advantages of anonymity in the first round with the conveniences of a group discussion prior to the second round, where all experts were given the chance to elaborate on their opinion. Given the often not observable or quantifiable differences in the disease outcome between serogroups found in studies, the guidance from this study may help to improve the understanding of the meningococcal disease burden in general, but may also do so by emphasizing MenB to detect potential differences in the disease outcome compared to other serogroups. Several limitations of this study should be acknowledged. Despite providing equal opportunities during the discussion, we cannot rule out that some arguments were prioritised over others. It is inherent to the DELPHI technique that the results will depend on the choice and composition of the expert panel and on how the questions were framed. In this sense, we note that the position of patient representatives was slightly underrepresented numbers-wise. To achieve appropriate framing of the questions, the questionnaire underwent quality control checks within the study group. Finally, the individual expert views and the degree of consensus among them could have changed 
between the time the DELPHI meeting took place and the time the study was written and reviewed. A longterm follow-up survey might be undertaken in some future study.

\section{Conclusion}

A better conceptualisation of the structure of IMD-specific sequelae and of how their diverse forms of severity might impact the QoL of survivors of IMD as well as their closest relatives and care-providers is needed to generate reliable and generalisable data on QoL in the future. The sought-after conceptualisation builds the basis to overcome the general lack of reliable and relevant measures of the health burden attributable to serogroup B IMD. However, the results of this DELPHI panel provide useful guidance on how to choose the study design, target population and appropriate QoL measures for future research. This will help promote the consistency in study methodology and sample characteristics.

\section{Additional file}

Additional file 1: The first-round DELPHI questionnaire. (DOCX 225 kb)

\section{Abbreviations}

CDC: Centers for Disease Control and Prevention; ECDC: European Centre for Disease Prevention and Control; HRQoL: Health-related quality of life; IMD: Invasive meningococcal disease; MenB: Serogroup B meningococcal; NICE: National Institute for Health and Care Excellence; QoL: Quality of life; UMV: Universal mass vaccination; WHO: World Health Organization

\section{Acknowledgements}

The authors would like to thank Hareth Al-Janabi, Robert Booy, and Paul Krabbe for their contribution to the study. They would also like to thank Business \& Decision Life Sciences platform for editorial assistance and manuscript coordination, on behalf of GSK. Marie Cloes coordinated manuscript development and provided editorial support. Geraldine Verplancke prepared and organised the DELPHI meeting.

\section{Funding}

GlaxoSmithKline Biologicals SA funded this study (GSK study identifiers: HO16-17940, HO-16-17947) and was involved in all stages of study conduct, including study design, analysis, and interpretation of the data. GlaxoSmithKline Biologicals SA also covered all costs associated with the development and publication of this manuscript.

\section{Availability of data and materials}

Not applicable.

\section{Authors' contributions}

$M B, H C, C D S, W G, M K i, F K, B L, O M, N V d V, R W$, and $C W$ were involved in the conception or the design of the study. CB, DB, HC, PDW, CDS, WG, PH, FK, $\mathrm{OM}, \mathrm{AML}, \mathrm{JPS}, \mathrm{NVdV}$, and $\mathrm{CW}$ participated in the collection or generation of the study data. DB, HC, PDW, CDS, WG, PH, MKn, FK, BL, OM, JPS, and RW were involved in the analysis or interpretation of the data. All authors participated in the development of this manuscript. All authors read and approved the final manuscript.

\section{Ethics approval and consent to participate}

Not applicable.

\section{Consent for publication}

Not applicable.

\section{Competing interests}

Monika Bullinger, Corinne Buysse, Wolfgang Greiner, Markus Knuf, Ole Marten, and Andrea Monteiro declare that they have no competing interests. Markus Kirchner, Nicolas Van de Velde, and Robert Welte are employees of the GSK group of companies. Florian Koerber was employed by the GSK group of companies at the time of study conduct. Nicolas Van de Velde and Robert Welte hold shares in the GSK group of companies. To support his research on the value of vaccination, David Bloom received grants from the Bill and Melinda Gates Foundation, the World Health Organization, GSK, Merck, Pfizer, Sanofi Pasteur, and Sanofi Pasteur-MSD. He also reports personal fees from GSK, Merck, Dynavax, and Pfizer, and participation in advisory boards for the same. He received personal fees for conducting research on the value of treatment from Gilead Life Sciences as well. Hannah Christensen is affiliated to the National Institute for Health Research Health Protection Research Unit (NIHR HPRU) in Evaluation of Interventions at University of Bristol in partnership with Public Health England (PHE). She reports monies paid to her employer from AstraZeneca, GSK, IMS Health, and Sanofi Pasteur. The views expressed are those of the author(s) and not necessarily those of the NHS, the NIHR, the Department of Health or Public Health England. Philippe De Wals received research grants, and reimbursements of travel expenses from vaccine manufacturers including GSK, Novartis, Sanofi Pasteur, and Pfizer, as well as from governmental agencies including the Quebec Ministry of Health and Social Services, Health Canada, and the Public Health Agency of Canada. Philippe De Wals has an advisory role as member of the Quebec immunization Committee and the (Canadian) National Advisory Committee on Immunization. Christian Dohna-Schwake reports a personal fee for GSK advisory board attendance. Philipp Henneke received reimbursements of travel expenses and honoraria from GSK, Pfizer and Abbvie, as well as from the Bill \& Melinda Gates Foundation and the WHO. Burkhard Lawrenz reports personal fees from GSK, during the conduct of the study, and personal fees from MSD, Sanofi, SPMSD, and Pfizer, outside the submitted work. JP Sevilla reports personal fees from GSK, during the conduct of the study, and outside the submitted work. He is employed by Data for Decisions LLC, an economics consulting firm which contracts to perform vaccine-related economic research for many pharmaceutical companies including but not limited to GSK $\mathrm{He}$ is also a Research Associate at the Harvard Chan School of Public Health (Chan School) where his research activities involve vaccine-related economic research supported by grants from pharmaceutical companies. At the Chan School, he is also a Technical Advisor on the Secretariat of the Value of Vaccines Research Network, which is funded by the Bill and Melinda Gates Foundation. Claire Wright is employed by the Meningitis Research Foundation (MRF), which has received educational grants from GSK and sponsorship for their biannual conference. She was also awarded a fee from GSK for contributing to an advisory board regarding this work. The consultancy fee was paid directly to MRF.

\section{Publisher's Note}

Springer Nature remains neutral with regard to jurisdictional claims in published maps and institutional affiliations.

\section{Author details}

${ }^{1}$ Department of Health Economics and Health Care Management, School of Public Health, Bielefeld University, Universitätsstr. 25, 33615 Bielefeld, Germany. ${ }^{2}$ GSK, Prinzregentenplatz 9, 81675 Munich, Germany. ${ }^{3}$ Department of Global Health and Population, Harvard T.H. Chan School of Public Health, 665 Huntington Avenue, Boston, Massachusetts 02115, USA. ${ }^{4}$ Department of Medical Psychology, University Medical Center Hamburg-Eppendorf, Martinistr. 52, 20246 Hamburg, Germany. ${ }^{5}$ Department of Pediatrics, Intensive Care Unit, Erasmus Medical Center, Sophia Children's Hospital, Wytemaweg 80, 3015, CN, Rotterdam, The Netherlands. ${ }^{6}$ Population Health Sciences, Bristol Medical School, University of Bristol, Oakfield House, Oakfield Grove, Bristol BS8 2BN, UK. ${ }^{7}$ Department of Social and Preventive Medicine, Laval University, Avenue de la Médecine 1050, Quebec City G1V 0A6, Canada. ${ }^{8}$ Pediatrics I, University Hospital of Essen, Hufelandstr. 55, 45147 Essen, Germany. ${ }^{9}$ Center for Pediatrics and Adolescent Medicine and Center for Chronic Immunodeficiency, Medical Center - University of Freiburg, 79106 Freiburg, Germany. ${ }^{10} \mathrm{HELIOS}$ Dr. Horst Schmidt Kliniken, Children's Hospital, Ludwig-Erhard-Str. 100, 65199 Wiesbaden, Germany. ${ }^{11}$ Pediatric Infectious Diseases, University Medicine, Mainz 55131, Germany. ${ }^{12}$ Association of Professional Pediatricians and Adolescent Medicine Specialists in Germany (Berufsverband der Kinder- und Jugendärzte e.V., BVKJ), Mielenforster Str. 2, 
51069 Köln, Germany. ${ }^{13}$ Private Practice for Pediatrics and Adolescent Medicine, Grafenstr. 80, 59821 Arnsberg, Germany. ${ }^{14}$ Asas Avaliacoes Economicas Em Saude Ltda ME, Rua Mario Pederneiras, 00055, APT 103 BLC II, Humaitá, Rio de Janeiro 22261-020, Brazil. ${ }^{15}$ Life Sciences Group, Data for Decisions, LLC, 681 Main Street, Suite 3-37, Waltham, Massachusetts 02451, USA. ${ }^{16}$ GSK, 20 Avenue Fleming, 1300 Wavre, Belgium. ${ }^{17}$ Meningitis Research Foundation, Newminster House, Baldwin Street, Bristol BS1 1LT, UK. ${ }^{18}$ Present Address: Institute of Health Economics and Health Care Management, Helmholtz Center Munich, German Research Center for Environmental Health $(\mathrm{GmbH})$, Ingolstädter Landstr. 1, 85764 Neuherberg, Germany. ${ }^{19}$ Present Address: Department of Pharmacy Systems, Outcomes and Policy, College of Pharmacy, University of Illinois at Chicago, Chicago, Illinois, USA.

\section{Received: 19 September 2018 Accepted: 13 May 2019} Published online: 22 May 2019

\section{References}

1. Strifler L, Morris SK, Dang V, Tu HA, Minhas RS, Jamieson FB, et al. The health burden of invasive meningococcal disease: a systematic review. J Pediatric Infec Dis Soc. 2016;5:417-30. https://doi.org/10.1093/jpids/piv065.

2. Al-Janabi H, Van Exel J, Brouwer W, Trotter C, Glennie L, Hannigan L, et al. Measuring health spillovers for economic evaluation: a case study in meningitis. Health Econ. 2016;25:1529-44. https://doi.org/10.1002/hec.3259.

3. Bhadhuri $\mathrm{A}$, Jowett $\mathrm{S}$, Jolly $\mathrm{K}, \mathrm{Al}$-Janabi $\mathrm{H}$. A comparison of the validity and responsiveness of the EQ-5D-5L and SF-6D for measuring health spillovers: a study of the family impact of meningitis. Med Decis Mak. 2017;37:882-93. https://doi.org/10.1177/0272989X17706355.

4. European Centre for Disease Prevention and Control (ECDC). Annual Epidemiological Report for 2016 - Invasive meningococcal disease. 2016. Available from: https://ecdc.europa.eu/sites/portal/files/documents/AER_for_ 2016-invasive-meningococcal-disease_1.pdf. Accessed 29 Mar 2019.

5. World Health Organization (WHO). Meningococcal meningitis - Key facts 2018. Available from: http://www.who.int/news-room/fact-sheets/detail/ meningococcal-meningitis. Accessed 17 Sept 2018.

6. Centers for Disease Control and Prevention (CDC). Epidemiology and Prevention of Vaccine-Preventable Diseases: Meningococcal Disease. Available from: https://www.cdc.gov/vaccines/pubs/pinkbook/mening.html. Accessed 3 Aug 2017.

7. Jafri RZ, Ali A, Messonnier NE, Tevi-Benissan C, Durrheim D, Eskola J, et al. Global epidemiology of invasive meningococcal disease. Popul Health Metrics. 2013;11:17. https://doi.org/10.1186/1478-7954-11-17.

8. Viner RM, Booy R, Johnson H, Edmunds WJ, Hudson L, Bedford H, et al. Outcomes of invasive meningococcal serogroup $B$ disease in children and adolescents (MOSAIC): a case-control study. Lancet Neurol. 2012;11:774-83. https://doi.org/10.1016/S1474-4422(12)70180-1.

9. Borrow R, Caugant DA, Ceyhan M, Christensen H, Dinleyici EC, Findlow J, et al. Meningococcal disease in the Middle East and Africa: findings and updates from the global meningococcal initiative. J Inf Secur. 2017;75:1-11. https://doi.org/10.1016/j.jinf.2017.04.007.

10. European Centre for Disease Prevention and Control (ECDC). Surveillance atlas of infectious diseases. Reported cases of invasive meningococcal disease in 2016. 2018. Available from: http://atlas.ecdc.europa.eu/public/ index.aspx. Accessed 24 July 2018.

11. Whittaker R, Dias JG, Ramliden M, Ködmön C, Economopoulou A, Beer N, et al. The epidemiology of invasive meningococcal disease in EU/EEA countries, 2004-2014. Vaccine. 2017;35:2034-41. https://doi.org/10.1016/j. vaccine.2017.03.007.

12. European Centre for Disease Prevention and Control (ECDC). Expert opinion on the introduction of the meningococcal B (4CMenB) vaccine in the EU/ EEA. 2017. Available from: http://ecdc.europa.eu/sites/portal/files/ documents/Introduction-of-4CMenB-vaccine.pdf. Accessed 23 Jan 2018.

13. Stoof SP, Rodenburg GD, Knol MJ, Rumke LW, Bovenkerk S, Berbers GA, et al. Disease burden of invasive meningococcal disease in the Netherlands between June 1999 and June 2011: a subjective role for serogroup and clonal complex. Clin Infect Dis. 2015;61:1281-92. https://doi.org/10.1093/cid/civ506.

14. Robert Koch Institute. Invasive Meningokokken-Erkrankungen 2012-2015 [Invasive meningococcal disease - cases 2012-2015]. Epidemiol Bull. 2016: 471-88. https://doi.org/10.17886/EpiBull-2016-064.2.

15. Gottfredsson M, Reynisson IK, Ingvarsson RF, Kristjansdottir H, Nardini MV, Sigurdsson JF, et al. Comparative long-term adverse effects elicited by invasive group B and C meningococcal infections. Clin Infect Dis. 2011;53: e117-24. https://doi.org/10.1093/cid/cir500.

16. Olbrich KJ, Müller D, Schumacher S, Beck E, Meszaros K, Koerber F. Systematic review of invasive meningococcal disease: sequelae and quality of life impact on patients and their caregivers. Infect Dis Ther. 2018;7:421-38. https://doi.org/ 10.1007/s40121-018-0213-2.

17. Wang B, Santoreneos R, Afzali H, Giles L, Marshall H. Costs of invasive meningococcal disease: a global systematic review. PharmacoEconomics. 2018;36:1201-22. https://doi.org/10.1007/s40273-018-0679-5.

18. Anonychuk A, Woo G, Vyse A, Demarteau N, Tricco AC. The cost and public health burden of invasive meningococcal disease outbreaks: a systematic review. PharmacoEconomics. 2013;31:563-76. https://doi.org/10.1007/s40273-013-0057-2.

19. Gasparini R, Landa P, Amicizia D, Icardi G, Ricciardi W, de Waure C, et al. Vaccinating Italian infants with a new multicomponent vaccine (Bexsero(R)) against meningococcal B disease: a cost-effectiveness analysis. Hum Vaccin Immunother. 2016;12:2148-61. https://doi.org/10.1080/21645515.2016.1160177.

20. Herdman M, Hoyle CK, Coles V, Carroll S, Devlin N. Assessing patient-reported outcomes in pediatric populations with vaccine-preventable infectious diseases: a systematic review of the literature (the PROCHID study). Value Health. 2016;19:109-19. https://doi.org/10.1016/j.jval.2015.10.001

21. Kreimeier S, Greiner W. EQ-5D-Y as a health-related quality of life instrument for children and adolescents: the Instrument's characteristics, development, current use, and challenges of developing its value set. Value Health. 2019; 22:31-7. https://doi.org/10.1016/j.jval.2018.11.001.

22. Sutherland JW. Architecting the future: a Delphi-based paradigm for normative system-building. In: Linstone HA, Turoff M, editors. The Delphi method: techniques and applications. Reading: Addison-Wesley; 1975.

23. Dalkey N, Helmer O. An experimental application of the DELPHI method to the use of experts. Manag Sci. 1962;9:458-67. https://doi.org/10.1287/mnsc.9.3.458.

24. Dalkey N. An experimental study of group opinion: the Delphi method. Futures. 1969;1:408-26. https://doi.org/10.1016/S0016-3287(69)80025-X.

25. Hsu C-C, Sandford BA. The Delphi technique: making sense of consensus. Pract Assess Res Eval. 2007;12 Available from: http://pareonline.net/getvn.asp? $v=12 \& n=10$.

26. Bowles N. The Delphi technique. Nurs Stand. 1999;13:32-6. https://doi.org/ 10.7748/ns1999.07.13.45.32.c2650

27. Efstathiou N, Ameen J, Coll AM. A Delphi study to identify healthcare users' priorities for cancer care in Greece. Eur J Oncol Nurs. 2008;12:362-71. https://doi.org/10.1016/j.ejon.2008.04.010.

28. Keeney S, Hasson F, McKenna HP. A critical review of the Delphi technique as a research methodology for nursing. Int J Nurs Stud. 2001;38:195-200. https://doi.org/10.1016/S0020-7489(00)00044-4.

29. Schmidt RC. Managing Delphi surveys using nonparametric statistical techniques. Decis Sci. 1997;28:763-74. https://doi.org/10.1111/j.1540-5915.1997.tb01330.x.

30. Kendall MG, Gibbons JD. Rank correlation methods. 5th ed. New York: Oxford Univ. Press; 1990

31. Alexandrov AV, Pullicino PM, Meslin EM, Norris JW. Agreement on diseasespecific criteria for do-not-resuscitate orders in acute stroke. Members of the Canadian and Western New York stroke consortiums. Stroke. 1996;27: 232-7. https://doi.org/10.1161/01.STR.27.2.232.

32. National Institute for Health and Care Excellence (NICE). Guide to the methods of technology appraisal 2013. 2013. Available from: https://www.nice.org.uk/ process/pmg9/chapter/the-reference-case. Accessed: 23 July 2018.

33. Wille N, Badia X, Bonsel G, Burstrom K, Cavrini G, Devlin N, et al. Development of the EQ-5D-Y: a child-friendly version of the EQ-5D. Qual Life Res. 2010;19:875-86. https://doi.org/10.1007/s11136-010-9648-y.

34. Stevens K. Developing a descriptive system for a new preference-based measure of health-related quality of life for children. Qual Life Res. 2009;18: 1105-13. https://doi.org/10.1007/s11136-009-9524-9.

35. Brooks R. EuroQol: the current state of play. Health Policy. 1996:37:53-72. https://doi.org/10.1016/0168-8510(96)00822-6. 